of quinine-that is to say, caused by a change of refrangibility in the ultra-violet rays. being a property of certain gases in the atmosphere. To possess this property the gas must be capable of absorbing either in part or entirely the ultra-violet and
violet rays, and of emitting them with a lowered resesses the property of absorption in the hichest degree in the ultra-violet region, and I have now to announce when seen in a glass bottle two inches in diameter
illuminated by an electric spark passing between cadmium electrodes. The color of the fluorescence is a observed in other cases but it is in the highest degree probable that oxygen is fluorescent, though this has
yet to be proved. There can be, however, little doubt
that the color of the sky is caused in part by the fluorescence of ozone, and also to some extent by the transnilssion of rays through the blue gas. The blue of
distance is doubtless to be attributed more to transdistance is doubtless to be attributed more to trans-
mission than the blue of the sky, though it is quite conceivable that fluorescence also here comes into play. Whatever other cause concurs in the production of the blue of the heavens, it has certainly been established
by M. Chappuis that the properties of ozone participate in its production.

In August, 1884, a very short note was sent by me to Nature concerning the red solar halo seen at Zerinatt
and on the Riffel berg with great distinctness. I recorded the occurrence of a dark band in the spectrum. slightly more refrangible than D, which was seen to vary in intensity; a second band a little less refrangible than
$\mathrm{D}$ was also observed. On account of the altitude at which the observations were made, viz., 9,000 feet, and the state of the weather at the time, these bands were considered to be due to some constituent of dry

The subject of the telluric rays has become of in creased interest since M. Cornu has studied the dark rays absorbed to which I refer: are both a little more and a little less refrangible than those figured on his map of this region. If we accept the number 5,890 value of the lines, $D^{1} \mathbf{D}^{2}$, the narrow bands observed by me have wave lengths about (1) 5,950 and (2) 5,770 at their darkest parts, as far as one can ascertain with a hand spectroscope giving excellent definition but smal
dispersion. They are very variable, being dependent dispersion. They are very variable, being dependent
on the state of the weather, and are more distinct and broader when viewed with the sun on the horizon. In (1) ent parts of the sky.
The less refrangible band, or broad line as it usuall appears, below $\mathrm{D}$, is generally overlapped by a band belonging to water vapor, the chief "rain band." On
this account observations at an elevation of 10,000 feet or so during perfectly dry weather were considered of interest. The bands were observed against the blue sky on several occasions, but they were also at other times entirely absent or barely visible. There is some lines being mistaken for the more refrangible band when it is not decidedly strong. Chappuis observe bands in the indication of the spectrum of ozone in the some atmosphere, but the reason why there must always be a difficulty in obtaining evidence of any absorption due to this substance arises from the strongest visible
band of ozone, with wave length 6,095 to 5,935 , being band of ozone, with wave length 6,095 to 5,935, being
masked by the band of water vapor; and secondly, be masked by the band of water vapor; and secondly, be the rays absorbed are only a small fraction of those due to absorption is either not seen or seen only with
difficulty. Owing to this fact we cannot distinguish difficulty. Owing to this fact we cannot distinguis bright; but when the sky is completely overoloude with cumuli, a faintly bluish tint is given to the clou only are the bright parts of the clouds blue, but their somewhat; at times it may be seen to shift about in the sky; it has been observed, for instance, to pass
over from southwest to northeast. The second but less conspicuous band of ozone absorbs rays with wav in a dry atırosphere at elevations varying from 6,000 to 10,000 feet, both in the blue of the sky and against white clouds. The measurements, very imperfectly made under difficulties, showed them to have wave
lengths about (1) 5,950, (2) 5,770 , in the center of the dark portion, while, according to Chappuis, the band

$$
\begin{array}{ll}
\text { (1) } 6,095 \text { to } 5,935 & \text { mean, } 6,010 \\
\text { (2) } 5,770 \text { to } 5,600 & \text { mean, } 5,680
\end{array}
$$

On Angstrom's chart, a dark band, diminishing in which is classed among the raies atmospheriques; 5,680 , this is similar to the band observed by me when viewing the sun or bright clouds riear

The work of Prof. Piazzi-Smyth, "Madeira Spectroscopic," does not give that portion of the spectrum
which" would serve for comparison. The "low sun band," $\delta$, comes very near to band (2), wave length
5,770 , while the "rain band " comes very near $(1), 6,095$. On several subsequent occasions the two bands were but with even less intensity Zermatt and the Riffe (November 10, 1884) that such an observation was reNo doubt a clear atmosphere, free from the turbidity so easily created by condensing moisture, is essential to frost in Central Russia. The measurements taken identified them with ozone bands, and leave scarcely
any doubt whatever of the presence of ozone in the atmosphere; and if it can be so recognized, it must
communicate its characteristic blue color to the air conmmunicate its characteristic blue color to the air
* Prof. Pickering has proved that sunlight as it reaches us is blue,
which mint be the case if it has passed through a blue medium (Proc.
American Academy of Sciences, 1880,
(Journ. Chem. Soc. Abstracts, vol. xlviii.. part 2, p. 713), close of 1883 proved highly favorable to such investiIn order to continue a series of observations on the
gitions. solar spectrum near D, it would be best to employ a
fairly good dispersion and large lenses with long focus still, to specially prepared photographic plates better The very extensive absorption of the ultra-violet
Thitive to the yellow rays. rays by oxygen leads us to expect it to be fluorescent.
All such absorbents are fluorescent more or less, and All such absorbents are fluorescent more or less, and venerally strongly, but when the absorbed rays are of
very short wave length, the fluorescence is not alway appear fluorescent by lime light nor by dull daylight but arestrongly so when seen by electric light,especially
if it has passed through no glass or other medium than if it has passed through no glass or other medium than stances are not fluorescent when seen in glass vessels, refrangibility would have been lowered by the fluorescent substance. In air, and by the light of an elec tric spark rich in ultra-violet rays, such as that from cadmium electrodes, almost every thing is fluorescent. The whole range of the cadmium spectrum has been white blotting paper. The light, of course, is feeble, and the eye has

Pure water, however, never appears fluorescent. Some solutions in water, which transmit all the ultra whether this is ar as 2,304, are fluorescent, though en decided.

It cannot any longer be doubted (1) that the extrem by the gases in the atmosphere, probably both by oxygen and ozone; (2) that the blue of the sky is a pheno menon caused by the fluorescence of the gaseous con-
stituents of the atmosphere, and probably ozone and oxygen are the chief fluorescent substances; (3) that ozone is generally present in the air in sufficient quan-
tity to render its characteristic absorption spectrum
visible, and that therefore it gives a blue color to the tmosphere by absorption, through which blue medium we observe distant views; (4) that water vapor doe onder like conditions and in the same manner as ozone
und Royal College of Science, Dublin. . N. HARTLEY.

\section{A SELECT METHOD OF PREPARING NITROGEN GAS.}

By H. N. Warren, Research Analyst.

THE formation of nitrogen gas when required as an quantities for research, always presents more or less

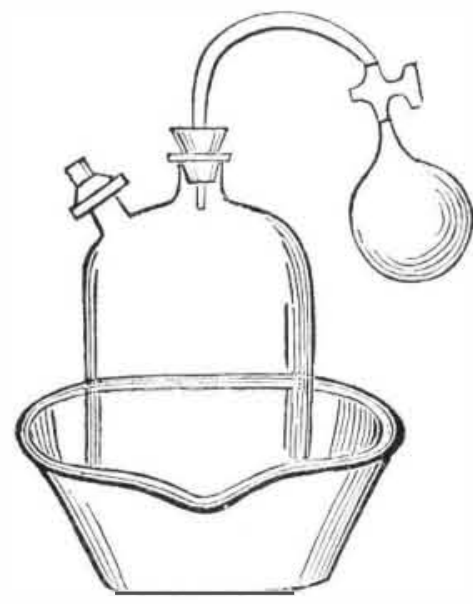

difficulty as regards its preparation; one process, still
recorded in the various hand-broks of science, being the action of nitric acid on raw meat. This metho may, however, without doubt be regarded as nothing
more than an alchemical memoir, sinceit would be evi dently difficult to state, or enumerate, the complicated same time would naturally contaminate the product. The action of potassium nitrite when heated in a suit able apparatus in admixture with ammonium chloride produces, when prepared cautiously, a moderately pure
gas, but owing to the inconstancy of the reaction the same is rendered considerably tedious and unprofitable. As a third instance, the reaction that phosphofor instance, when confined by the aid of a bell jar and in direct contact with water, at once presents an paring a very pure gas, and direct from the atmospher This, although a favorite method of most demonstrat-
ors and others when experimenting with or illustrating the component parts of the atmosphere, yet at the same time few, if any, seem to have adopted a plan to recover, or more correctly to collect, the so prepare gas, which by a slight alteration in the form of appa-
ratus to that generally employed may be procured in any desired quantity.

The accompanying figure, which is intended to illusdoubly tubulated gas receiver or bell jar connected either with a bladder provided with a suitable stop cock, or, if required in larger quantities, to an ordinaof gas holder, the outer vessel containing a sufficiency he action to be immersed to the required height. In using the apparatus, all that is necessary is to apply a hot wire to the pieces of phosphorus contained in a
small brass dish, the same being allowed to float upon the surface of the water. This is most readily accomplished by withdrawing the stopper from the side tubule, inserting a hot wire in order to start the com-
bustion of the phosphorus, and replacing the stopper. The apparatus and its contents. after the combustion of the phosphorus has terminated, is allowed order to allow the fumes of phosphoric pentoxide oc-
casioned by the combustion of the phosphorus to unite with the water. The stop cock, in connection
with the receiver intended for the reception of the gas, is now opened, the weight of the bell jar exerting a pressure sufficient to allow of its contents to be dis-
charged into the receiver. The tap in connection with flo side tubule again withdrawn, thus allowing, on raising the apparatus to the surface of the water, to admit of a
resh supply of air, which may be proceeded with as before. By this method, operating with a jar capable
of retaining one cubic foot of atmosphere, a gas holder able to contain six cubic feet of nitrogen may be filled AN AUTOMATIC WASH BOTTLE. By J. F. Jonks.

THe figure represents a section through the flask,

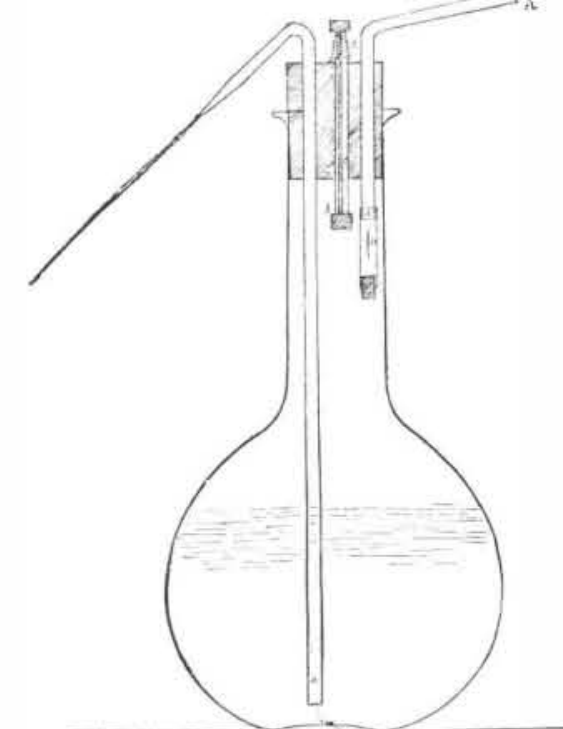

ordinary wash bottle, with the exception of two valves. One of these is placed at the end of the air tube, $a$.
It is a "Bunsen valve," which consists of a piece of rubber tubing, placed over the end of the glass tube. piece of glass rod. In this rubber tube there is a slit
cut, which will allow air to pass into the flask, but will
not allow it to pass out. The pressure of the air is not allow it to pass out. The pressure of the air is only be restored by water flowing out through the delivery tube. In order that this flow may be stopped
when desired, the other valve is placed in. It consists of a glass tube, $c$ into which a small wooden rod is fits over the end of the glass tube, and to make it fit air tight, a rubber washer is placed at $d$. This disk is held tight against the tube by a small spiral spring, $\mathrm{E}$.
After blowing air in at A, and causing water to flow from the delivery tube, equilibrium can be at once reing on the button, $f$. It is a stopped, by simply pressapparatus, as it saves the trouble of blowing during the whole time of washing a precipitate. By applying

PRODUCING ALUMINUM AND OTHER METALS AND ALLOYS.

THIs invention relates to apparatus for producing, refractory compounds. B is a series of carbon plates forming the positive pole, the negative pole being molbox. The crucible is of carbon. Copper pins, $a^{1}$, con-
duct the current to interior of crucible, A. The positive electrode, B, dips into crucible, A. $g$ is a clamp
for holding the plates, $b$, and by means of which the electrode can be raised or lowered. The crucible, $A$, formed openings, $n$, for charging the material to be formed openings, n, for charging the material to be
treated, which also serve as outlet passages for the
gases produced. Plates, $o$ each having a handle, $o^{2}$, gases produced. Plates, $o$, each having a handle, $o^{2}$,
close the holes during the melting process. At the

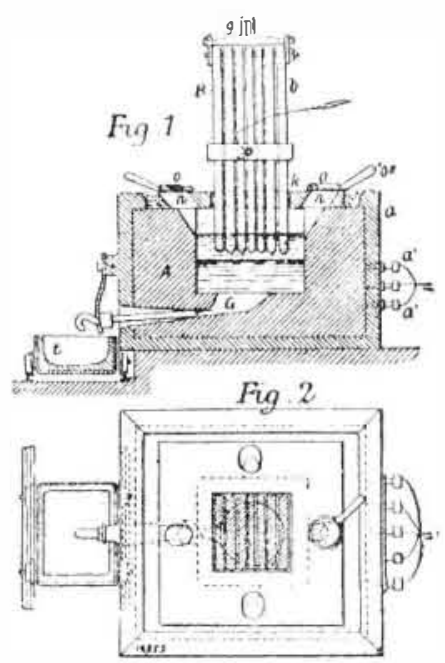

commencement of the operation the electrode. B, is in crucible, A. a current is passed through and melts the copper which serves as the negative electrode, upo

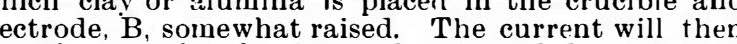
pass through the alumina and melt and decompose 